\title{
Timing in the surgical evacuation of spinal epidural abscesses
}

\author{
George M. Ghobrial, M.D., Sara Beygi, M.D., Matthew J. Viereck, B.S., \\ Christopher M. Maulucci, M.D., Ashwini Sharan, M.D., Joshua Heller, M.D., \\ Jack Jallo, M.D., Ph.D., Srinivas Prasad, M.D., and James S. Harrop, M.D.
}

Department of Neurological Surgery, Thomas Jefferson University Hospital, Philadelphia, Pennsylvania

\begin{abstract}
Object. One often overlooked aspect of spinal epidural abscesses (SEAs) is the timing of surgical management. Limited evidence is available correlating earlier intervention with outcomes. Spinal epidural abscesses, once a rare diagnosis carrying a poor prognosis, are steadily becoming more common, with one recent inpatient meta-analysis citing an approximate incidence of 1 in 10,000 admissions with a mortality approaching $16 \%$. One key issue of contention is the benefit of rapid surgical management of SEA to maximize outcomes. Timing of surgical management is definitely one overlooked aspect of care in spinal infections. Therefore, the authors performed a retrospective analysis in which they evaluated patients who underwent early (evacuation within 24 hours) versus delayed surgical intervention ( $>24$ hours) from the point of diagnosis, in an attempt to test the hypothesis that earlier surgery results in improved outcomes.

Methods. A retrospective review of a prospectively maintained adult neurosurgical database from 2009 to 2011 was conducted for patients with the diagnostic heading: epidural abscess, infection, osteomyelitis, osteodiscitis, spondylodiscitis, and abscess. The primary end point for each patient was neurological grade, measured as an American Spinal Injury Association Impairment Scale grade using hospital inpatient records on admission and discharge. Patients were divided into early surgical $(<24$ hours) and delayed surgical cohorts.

Results. Eighty-seven consecutive patients were identified ( 25 females; mean age 55.5 years, age range 18-87 years). Fifty-four patients received surgery within 24 hours of admission (mean time from admission to incision, 11.2 hours), and 33 underwent surgery longer than 24 hours (mean 59 hours) after admission. Of the 54 patients undergoing early surgery $45(85 \%)$ had a neurological deficit, whereas in the delayed surgical group $21(64 \%)$ of 33 patients presented with a neurological deficit $(\mathrm{p}=0.09)$. Patients in the delayed surgery cohort were significantly older by 10 years (59.6 vs 51.8 years, $\mathrm{p}=0.01)$. With regard to history of prior revision, body mass index, intravenous drug abuse, tobacco use, prior radiation therapy, diabetes, chronic systemic infection, and prior osteomyelitis, there were no significant differences. There was no significant difference between early and delayed surgery groups in neurological grade on presentation, discharge, or location of epidural abscess. The most common organism isolated was Staphylococcus aureus $(\mathrm{n}=51,59.3 \%)$. The incidence of methicillin-resistant S. aureus was $21 \%(18$ of 87$)$.

Conclusions. Evacuation within 24 hours appeared to have a relative advantage over delayed surgery with regard to discharge neurological grade. However, due to a limited, variable sample size, a significant benefit could not be shown. Further subgroup analyses with larger populations are required. (http://thejns.org/doi/abs/10.3171/2014.6.FOCUS14120)
\end{abstract}

KEY Words
osteodiscitis

$\mathrm{S}$ PINAL epidural abscesses (SEAs), once a rare diagnosis carrying a poor prognosis, ${ }^{4,20}$ are steadily becoming more common, with a recent inpatient meta-analysis citing an approximate incidence of 1 in 10,000 admissions ${ }^{4,14}$ Once thought to be widely fatal, ${ }^{4}$ the mortality of SEA has been reportedly as high as $16 \% .{ }^{15-17}$ Further contributing to the morbidity is the rising prevalence of highly virulent organisms such as methicillin-resistant

\footnotetext{
Abbreviations used in this paper: AIS = American Spinal Injury Association Impairment Scale; MRSA = methicillin-resistant Staphylococcus aureus; SEA = spinal epidural abscess.
}

Staphylococcus aureus (MRSA), attributed to the rise in intravenous drug abuse, chronic antibiotic use, and hemodialysis. ${ }^{1}$ Two key issues of contention have been the need for early surgical management of an SEA to maximize outcomes and the benefit of surgical over medical management in the form of intravenous antibiotics. ${ }^{1-3,15,18,19}$ Recently, relatively large published retrospective series have found no additional benefit added by early surgical evacuation to antibiotic treatment. ${ }^{1,10}$ However, early surgical management of cervical and thoracic epidural abscesses continues to be common practice, especially in the face of progressive neurological deterioration. 


\section{G. M. Ghobrial et al.}

Timing of surgical management is one overlooked aspect of care in spinal infections. Limited prospective data are available regarding the management of this disease. The presence of a purulent bacterial empyema or phlegmon in the epidural space overlying the spinal cord is considered as a surgical emergency. This perceived risk is largely due to pathological studies demonstrating diffuse thrombosis of the underlying vasculature in rabbits, published almost 30 years ago. ${ }^{6-8}$ In the present study, we present a large surgical series of spinal decompression for SEA in the adult population, in an attempt to test the hypothesis that earlier surgery correlates with improved outcomes. ${ }^{1}$

\section{Methods}

\section{Study Selection Criteria}

A retrospective review of a prospectively maintained neurosurgical database from 2009 to 2011 was conducted for all consecutive adult patients undergoing surgical treatment of an epidural abscess. The ICD-9-CM code 324.1 was queried in an attempt to encounter prior cases involving abscesses of the spinal canal. To obtain all consecutive patients in this database, all additional patients with the following diagnostic headings and/or descriptions were included: epidural abscess, infection, osteomyelitis, osteodiscitis, spondylodiscitis, and abscess. All patients without admission and discharge neurological examination records, admission MRI, and positive bacterial cultures confirming infection were excluded.

\section{Diagnosis}

Early diagnosis in all cases of SEA was made first by clinical suspicion followed by rapid acquisition of a contrasted MRI scan. Radiographic confirmation by contrastadministered T1-weighted MRI of epidural abscess was noted for all patients. Supporting clinical evidence such as $\mathrm{C}$-reactive protein, erythrocyte sedimentation rate, and white blood cell counts are routinely obtained throughout the course of admission. The duration to surgery was measured from the point of MRI confirmation of SEA to incision by hospital records, when available.

\section{Neurological Presentation}

The American Spinal Injury Association Impairment Scale (AIS) ${ }^{12}$ grade was calculated from hospital inpatient records at the immediate time of preoperative neurosurgical consultation and discharge. Numerical scores of 1 through 5 were assigned to grades A through $\mathrm{E}$, respectively. Patients were then divided into 2 cohorts using a 24-hour cutoff into early ( $<24$ hours) and delayed ( $>24$ hours) surgical cohorts. Baseline characteristics, risk factors for infection, and admission neurological function were recorded.

\section{Statistical Analysis}

Nonparametric data were comparatively assessed using a Wilcoxon signed-rank test. Fisher's exact test, and ANOVA were carried out using a statistical software package (version 8.00, JMP software, SAS Institute).

\section{Results}

Eighty-seven consecutive patients were identified (25 females; mean age $55.5 \pm 13.6$ years [SD], age range 1887 years). Fifty-four patients had received surgery within 24 hours of admission (mean time from admission to incision, 11.2 hours), and 33 had surgery longer than 24 hours (mean 59 hours) after admission. Patients in the delayed surgery cohort were significantly older by 10 years $(59.6$ vs 51.8 years, $\mathrm{p}=0.01$ ). With regard to history of prior spinal surgery, body mass index, intravenous drug abuse, tobacco use, prior radiation therapy, diabetes, chronic systemic infection, and prior known osteomyelitis, there were no significant differences (Table 1).

\section{Surgical Characteristics}

Sixty-nine (79\%) of all SEAs were predominantly cervical or thoracic. There were more than twice as many cervical SEAs treated within 24 hours versus after the cutoff point ( 27 vs $12, p=0.08)$. Forty-nine patients $(56.3 \%)$ underwent a combined anterior/posterior approach, 30 (34.1\%) underwent a dorsal approach only, and 8 underwent a ventral approach $(9.1 \%)$. Sixty-three percent of the ventral-only approaches $(5 / 8)$ were for ventrally located cervical epidural abscesses. The most common organism cultured was methicillin-sensitive Staphylococcus aureus (53\%), followed by MRSA (27\%) (Table 2). In one case, both Coryneform spp. and Candida albicans were cultured.

\section{Neurological Outcomes}

A higher proportion of patients in the early surgical cohort had a neurological deficit (AIS Grade A-D, 45/54, $83 \%)$ than in the delayed surgery cohort $(21 / 33,64 \%, p=$ 0.09 ) (Table 3). The mean improvement between the early and delayed surgical groups was 0.41 and 0.39 , respectively, which did not differ significantly (Table 4). The location of the epidural abscess and AIS grade did not vary significantly between cohorts (Table 5). All patients who underwent early surgery either remained the same or improved (Table 6). There were no instances of immediate postoperative neurological worsening. One AIS Grade A patient in the early surgery cohort made a full recovery. The other 5 patients in the AIS Grade A group remained unchanged by the time of discharge. In the delayed surgery cohort, 4 patients classified as AIS Grade D deteriorated to AIS Grade $\mathrm{C}$ on discharge (Table 7), with 6 remaining unchanged and 3 improving. Most patients demonstrated improvement in neurological grade after surgery (Table 6), with only 1 case of neurological worsening at time of discharge (Table 7).

\section{Cord Signal Intensity}

The rate of T2 hyperintensity was $50 \%$ on MRI (18 of 36) for the cervical spine. The mean AIS score for patients with T2 signal hyperintensity was 3.6 with a mean improvement of 0.5 by discharge. The mean AIS grade was 4.17 in the $\mathrm{T} 2$ hyperintensity negative group with a mean improvement of 0.24 . While the initial examination was worse in the T2 hyperintensity group, as expected, the recovery was unexpectedly twice as great in the cord 
Timing in the surgical evacuation of spinal epidural abscesses

TABLE 1: Baseline characteristics*

\begin{tabular}{|c|c|c|c|}
\hline \multirow[b]{2}{*}{ Parameter } & \multicolumn{2}{|c|}{ Value† } & \multirow[b]{2}{*}{$\mathrm{p}$ Value } \\
\hline & $\begin{array}{c}\text { Early } \\
\text { Intervention }\end{array}$ & $\begin{array}{l}\text { Delayed } \\
\text { Intervention }\end{array}$ & \\
\hline time to surgical evacuation & $<24 \mathrm{hrs}$ & $>24 \mathrm{hrs}$ & \\
\hline no. of patients & 54 & 33 & \\
\hline no. of females & 11 & 14 & 0.14 \\
\hline mean age in yrs & 51.8 & 59.6 & 0.01 \\
\hline prior revision & 3 & 7 & 0.23 \\
\hline prior osteomyelitis on antibiotics & 3 & 9 & 0.09 \\
\hline tobacco use & 12 & 8 & 0.90 \\
\hline intravenous drug abuse & 16 & 7 & 0.58 \\
\hline BMI & 27.7 & 28.0 & 0.78 \\
\hline prior radiation & 2 & 0 & 0.77 \\
\hline diabetes & 13 & 11 & 0.50 \\
\hline $\begin{array}{l}\text { prior known systemic infection } \\
\text { infection by region }\end{array}$ & 21 & 9 & 0.57 \\
\hline cervical & 27 & 12 & 0.08 \\
\hline thoracic & 19 & 11 & 0.82 \\
\hline lumbar & 8 & 10 & 0.11 \\
\hline
\end{tabular}

* $\mathrm{BMI}=$ body mass index.

$\dagger$ Values are number of patients unless noted otherwise.

signal group (0.50 vs 0.24$)$. However, the results were not statistically significant $(\mathrm{p}=0.11)$.

\section{Discussion}

\section{Timing in Surgical Evacuation}

The acuity of surgical evacuation of an SEA remains a contentious issue. Retrospective data provide the majority of guidance. In a prior operative series by Connor et al., ${ }^{2}$ using a 3-day cutoff, a larger proportion of patients demonstrated improvement in the early group relative to delayed surgery ( $>3$ days). The study by Connor et al., however, highlights the bias commonly seen in retrospective series, which predominantly favored early surgery (38 [67\%] of 57 patients). In this study, demonstration of neurological improvement did not reach statistical significance in the early operative group. Further overshadowing the results, the mean time to surgery was twice as long in

TABLE 2: Causative organism

\begin{tabular}{lc}
\hline \multicolumn{1}{c}{ Organism } & No. of Patients (\%) \\
\hline methicillin-sensitive S. aureus & $47(53)$ \\
MRSA & $24(27)$ \\
coagulase-negative S. aureus & $3(3)$ \\
Streptococcus spp. & $3(3)$ \\
Pseudomonas aeruginosa & $3(3)$ \\
Escherichia coli & $3(3)$ \\
Coryneform spp. & $2(2)$ \\
Candida albicans & $2(2)$ \\
polymicrobial & $1(1)$ \\
\hline
\end{tabular}

TABLE 3: Neurological grade at time of admission

\begin{tabular}{cccc}
\hline & \multicolumn{2}{c}{ No. of Patients } & \\
\cline { 2 - 3 } AIS Grade & $<24$ Hrs & $>24$ Hrs & p Value \\
\hline A & 3 & 3 & 0.79 \\
B & 5 & 3 & 0.62 \\
C & 9 & 3 & 0.45 \\
D & 26 & 13 & 0.45 \\
E & 11 & 11 & 0.11 \\
\hline
\end{tabular}

the group with superior neurological recovery (6.3 vs 3.2 days).

Patel and colleagues retrospectively reviewed surgical versus nonsurgical management of SEAs in 128 patients with the goal of finding risk factors for poor neurological outcomes. ${ }^{13}$ In their study, they found that antibiotic therapy failed in a large portion of patients $(n=21,41 \%)$, and ultimately these patients received surgical management. They support the finding that earlier surgery is associated with improved neurological functioning.

The high rate of failure reported by Patel et al. with an initial course of modern-day broad-spectrum antibiotics is not an uncommon finding. ${ }^{13} \mathrm{Kim}$ et al. found a 99\% probability of antibiotic failure with patients presenting with an age of older than 65 years, diabetes, MRSA, or neurological compromise. ${ }^{11}$

In our series, early surgery appeared to offer a benefit in all patients presenting with a neurological deficit, despite not reaching statistical significance. One AIS Grade A patient showed dramatic improvement to AIS Grade E, 4 patients improved from AIS Grade B to Grade C, 78\% of AIS Grade C (7/9) patients improved to AIS Grade D, and $50 \%$ of AIS Grade D patients improved to AIS Grade E on discharge (Table 6). Delayed surgery showed modest improvement in AIS Grade B and C subgroups. Four AIS Grade D patients worsened to AIS Grade C, while 3 patients improved to AIS Grade E. While the overall trend appears to favor early surgery, the sample was too small to demonstrate a significant effect.

\section{Limitations}

This study suffers from several limitations specifically due to its retrospective design. Due to the often nebulous presenting signs and symptoms of SEAs, the variable

\section{TABLE 4: Neurological grade at time of discharge*}

\begin{tabular}{|c|c|c|c|}
\hline AIS Grade & $<24 \mathrm{Hrs}$ & $>24 \mathrm{Hrs}$ & $\mathrm{p}$ Value \\
\hline A & 2 & 3 & 0.98 \\
\hline B & 1 & 0 & 0.89 \\
\hline C & 6 & 3 & 0.87 \\
\hline D & 20 & 13 & 0.42 \\
\hline E & 25 & 11 & 0.27 \\
\hline improvement (raw score/no. of patients) $\dagger$ & +0.41 & +0.39 & 0.17 \\
\hline
\end{tabular}

* Values are number of patients unless indicated otherwise. Three patients did not undergo examination at discharge.

† 1 = AIS Grade A (complete injury), 5 = AIS Grade E (neurologically intact). 
TABLE 5: Admission neurological grade by region*

\begin{tabular}{|c|c|c|c|c|c|c|c|c|}
\hline \multirow[b]{2}{*}{ Parameter } & \multicolumn{4}{|c|}{ Early Intervention (<24 hrs) } & \multicolumn{4}{|c|}{ Delayed Intervention (>24 hrs) } \\
\hline & Cervical & Thoracic & Lumbar & p Value $†$ & Cervical & Thoracic & Lumbar & p Value \\
\hline AIS grade & & & & 0.20 & & & & 0.76 \\
\hline$A$ & 0 & 2 & 1 & & 2 & 1 & 0 & \\
\hline B & 2 & 3 & 0 & & 1 & 2 & 0 & \\
\hline C & 4 & 5 & 0 & & 1 & 0 & 2 & \\
\hline$D$ & 14 & 5 & 7 & & 3 & 5 & 6 & \\
\hline$E$ & 7 & 4 & 0 & & 5 & 3 & 2 & \\
\hline mean improvementf & +0.48 & +0.45 & +0.29 & 0.86 & +0.111 & +0.41 & +0.30 & 0.79 \\
\hline
\end{tabular}

presentation makes a true measurement of early surgical evacuation difficult. Furthermore, the time frequently spent by an emergency or inpatient medicine service in routine admission and workup was variable and frequently resulted in extended time periods before an MRI could be obtained for diagnosis of an SEA.

The classic diagnosis of SEA with spinal mechanical pain and tenderness, fever, and neurological abnormalities is likely only sufficient to capture a maximum of $10 \%$ of patients, according to a study by Davis and colleagues. ${ }^{5}$ As a result, in this study, the time to surgery was measured from the point of MRI confirmation, irrespective of the onset of prior symptoms. Here, we measured early versus delayed as the time from MRI diagnosis to incision. In the case of lumbar SEA, some of these infections were not ad-

TABLE 6: Surgery within 24 hours: neurological examination on discharge*

\begin{tabular}{crrrrr}
\hline & \multicolumn{5}{c}{ AIS Grade on Discharge } \\
\cline { 2 - 6 } Presenting AIS Grade & A & B & C & D & E \\
\hline A & 2 & 0 & 0 & 0 & 1 \\
B & 0 & 1 & 4 & 0 & 0 \\
C & 0 & 0 & 2 & 7 & 0 \\
D & 0 & 0 & 0 & 13 & 13 \\
E & 0 & 0 & 0 & 0 & 11 \\
\hline
\end{tabular}

* Values are number of patients.

TABLE 7: Surgery after 24 hours: neurological examination on discharge*

\begin{tabular}{crrrrr}
\hline & \multicolumn{5}{c}{ AIS Grade on Discharge } \\
\cline { 2 - 6 } Presenting AIS Grade & A & B & C & D & E \\
\hline A & 3 & 0 & 0 & 0 & 0 \\
B & 0 & 0 & 0 & 1 & 2 \\
C & 0 & 0 & 2 & 1 & 0 \\
D & 0 & 0 & 4 & 6 & 3 \\
E & 0 & 0 & 0 & 0 & 11 \\
\hline
\end{tabular}

* Values are number of patients. jacent to the spinal cord and therefore were not considered emergencies unless there was a neurological deficit. While there were 11 lumbar SEAs treated longer than 24 hours after MRI acquisition versus 7 in the early surgery group, the groups were not significantly different (Table 2).

Due to the retrospective design of this study, it is difficult to say whether a higher proportion of cervical and thoracic AIS Grade B, C, and D patients improved by 1 grade due to the earlier surgical intervention or because of a variable response of lumbar SEA to surgical intervention. An attempt was made to show that the baseline clinical characteristics were fairly similar (Table 1). However, the delayed surgery group comprised a significantly older patient population (59.6 vs $51.8, \mathrm{p}=0.01$ ), which could easily explain the difference in outcomes as age has been shown to be a poor prognostic factor in SEA surgical outcomes. ${ }^{1,2,9}$ Lastly, lack of long-term surveillance limits our conclusions regarding efficacy.

\section{Conclusions}

Based on the data available, evacuation within 24 hours of MRI identification of a spinal epidural abscess does not demonstrate a statistically significant benefit over delayed surgery with regard to discharge neurological grade. Although there are trends suggesting benefit in specific subgroups, these do not reach statistical significance. Further subgroup analysis with larger populations is required for more detailed analysis.

\section{Disclosure}

The authors report no conflict of interest concerning the materials or methods used in this study or the findings specified in this paper.

Author contributions to the study and manuscript preparation include the following. Conception and design: Ghobrial. Acquisition of data: Ghobrial, Beygi, Viereck. Analysis and interpretation of data: Maulucci. Drafting the article: Ghobrial, Beygi. Critically revising the article: Harrop, Maulucci. Reviewed submitted version of manuscript: Harrop, Sharan, Heller, Jallo, Prasad. Approved the final version of the manuscript on behalf of all authors: Harrop.

\section{References}

1. Adogwa O, Karikari IO, Carr KR, Krucoff M, Ajay D, Fatemi 


\section{Timing in the surgical evacuation of spinal epidural abscesses}

P, et al: Spontaneous spinal epidural abscess in patients 50 years of age and older: a 15-year institutional perspective and review of the literature. Clinical article. J Neurosurg Spine 20:344-349, 2014

2. Connor DE Jr, Chittiboina P, Caldito G, Nanda A: Comparison of operative and nonoperative management of spinal epidural abscess: a retrospective review of clinical and laboratory predictors of neurological outcome. Clinical article. J Neurosurg Spine 19:119-127, 2013

3. Curry WT Jr, Hoh BL, Amin-Hanjani S, Eskandar EN: Spinal epidural abscess: clinical presentation, management, and outcome. Surg Neurol 63:364-371, 2005

4. Darouiche RO: Spinal epidural abscess. N Engl J Med 355: 2012-2020, 2006

5. Davis DP, Wold RM, Patel RJ, Tran AJ, Tokhi RN, Chan TC, et al: The clinical presentation and impact of diagnostic delays on emergency department patients with spinal epidural abscess. J Emerg Med 26:285-291, 2004

6. Feldenzer JA, McKeever PE, Schaberg DR, Campbell JA, Hoff JT: Experimental spinal epidural abscess: a pathophysiological model in the rabbit. Neurosurgery 20:859-867, 1987

7. Feldenzer JA, McKeever PE, Schaberg DR, Campbell JA, Hoff JT: The pathogenesis of spinal epidural abscess: microangiographic studies in an experimental model. J Neurosurg 69:110-114, 1988

8. Feldenzer JA, Waters DC, Knake JE, Hoff JT: Anterior cervical epidural abscess: the use of intraoperative spinal sonography. Surg Neurol 25:105-108, 1986

9. Huang PY, Chen SF, Chang WN, Lu CH, Chuang YC, Tsai NW, et al: Spinal epidural abscess in adults caused by Staphylococcus aureus: clinical characteristics and prognostic factors. Clin Neurol Neurosurg 114:572-576, 2012

10. Karikari IO, Powers CJ, Reynolds RM, Mehta AI, Isaacs RE: Management of a spontaneous spinal epidural abscess: a single-center 10-year experience. Neurosurgery 65:919-924, 2009

11. Kim SD, Melikian R, Ju KL, Zurakowski D, Wood KB, Bono $\mathrm{CM}$, et al: Independent predictors of failure of nonopera- tive management of spinal epidural abscesses. Spine $\mathbf{J}$ [epub ahead of print], 2013

12. Marino RJ, Graves DE: Metric properties of the ASIA motor score: subscales improve correlation with functional activities. Arch Phys Med Rehabil 85:1804-1810, 2004

13. Patel AR, Alton TB, Bransford RJ, Lee MJ, Bellabarba CB, Chapman JR: Spinal epidural abscesses: risk factors, medical versus surgical management, a retrospective review of 128 cases. Spine J 14:326-330, 2014

14. Reihsaus E, Waldbaur H, Seeling W: Spinal epidural abscess: a meta-analysis of 915 patients. Neurosurg Rev 23:175-205, 2000

15. Rigamonti D, Liem L, Sampath P, Knoller N, Namaguchi Y, Schreibman DL, et al: Spinal epidural abscess: contemporary trends in etiology, evaluation, and management. Surg Neurol 52:189-197, 1999

16. Rigamonti D, Metellus P: Spinal epidural abscess. N Engl J Med 356:638-639, 2007 (Letter)

17. Sampath P, Rigamonti D: Spinal epidural abscess: a review of epidemiology, diagnosis, and treatment. J Spinal Disord 12: 89-93, 1999

18. Savage K, Holtom PD, Zalavras CG: Spinal epidural abscess: early clinical outcome in patients treated medically. Clin Orthop Relat Res 439:56-60, 2005

19. Siddiq F, Chowfin A, Tight R, Sahmoun AE, Smego RA Jr: Medical vs surgical management of spinal epidural abscess. Arch Intern Med 164:2409-2412, 2004

20. Tompkins M, Panuncialman I, Lucas P, Palumbo M: Spinal epidural abscess. J Emerg Med 39:384-390, 2010

Manuscript submitted April 9, 2014.

Accepted June 4, 2014.

Please include this information when citing this paper: DOI: 10.3171/2014.6.FOCUS14120.

Address correspondence to: James S. Harrop, M.D., Department of Neurological Surgery, Thomas Jefferson University Hospital, 909 Walnut St.,Philadelphia,PA 19107.email: james.harrop@jefferson. edu. 